\title{
Strates
}

STRATES Matériaux pour la recherche en sciences sociales

$12 \mid 2006$

Nouvelles tensions impériales et recompositions en Europe centrale, orientale, et CEI

\section{La fin du communisme}

Jean-Yves Potel

\section{(2) OpenEdition}

1 Journals

\section{Édition électronique}

URL : http://journals.openedition.org/strates/1612

DOI : $10.4000 /$ strates. 1612

ISSN : $1777-5442$

Éditeur

Laboratoire Ladyss

\section{Édition imprimée}

Date de publication : 31 décembre 2006

ISSN : 0768-8067

Référence électronique

Jean-Yves Potel, « La fin du communisme », Strates [En ligne], 12 | 2006, mis en ligne le 19 juillet 2007, consulté le 08 septembre 2020. URL : http://journals.openedition.org/strates/1612 ; DOI : https:// doi.org/10.4000/strates. 1612

Ce document a été généré automatiquement le 8 septembre 2020.

Tous droits réservés 


\title{
La fin du communisme
}

\author{
Jean-Yves Potel
}

1 Plus de dix-sept ans après, 1989 prend une portée historique de plus grande envergure que celle envisagée à l'époque. L'effondrement de l'empire soviétique est à l'origine d'au moins trois grands bouleversements politiques en Europe et dans le monde. D'abord, l'apparition de nouveaux États démocratiques. Ce n'est plus aujourd'hui contesté, même si les critères d'appréciation sont en discussion. L'existence de ces nouveaux États renvoie immédiatement à la perspective d'une nouvelle unification européenne.

2 Ensuite, nous avons assisté à la formation en Europe de petits régimes autoritaires aux prétentions territoriales qui, soit ont voulu s'étendre en déstructurant un ensemble plus large, soit se sont repliés vers un espace plus limité. Ces régimes ont été responsables de guerres (réintroduisant le crime contre l'humanité) ou de tensions et d'atteintes aux droits de l'homme qui ont déstabilisé la région. Mais ils ne sont pas parvenus à s'installer durablement. Leur participation (volontaire ou non) à un système régional d'États, dominé par l'Union européenne, les a contraints à changer de voie (Slovaquie, Roumanie, Albanie, Croatie...) ou à démissionner (Serbie).

3 Enfin, le système de relations internationales entre États a été complètement bouleversé. Les spécialistes de la discipline évoquent la fin du "modèle de Wesphalie ", c'est-à-dire d'un système de défense et de sécurité, fondé sur la souveraineté des États nationaux. Il garantissait, depuis la fin de la guerre de Trente ans (1648), «les notions de non-interférence, d'égalité de principe, de respect des frontières et de réciprocité entre les États ». L'après " guerre froide » et la mondialisation mettent en question ce modèle, du moins dans le domaine de la sécurité1. Ce qui suscite de nombreux débats sur l'ampleur du changement. Certains y voient une « crise transitoire des capacités de l'État» qui devrait s'adapter, d'autres pensent au contraire qu'une "gouvernance globale » doit être mieux pensée devant l'incapacité croissante de l'État national à prendre en charge les nouveaux défis. Mais, dans tous les cas, ce bouleversement politique pose la question de «l'intégration régionale des États » et du "juste équilibre entre un "souverainisme" devenu utopique et un "mondialisme" subordonnant totalement l'État au libéralisme du marché ${ }^{~}$ ". 
4 Ces trois bouleversements politiques, dont nous pouvons commencer à mesurer aujourd'hui les immenses conséquences, n'ont été possibles qu'avec la fin du système communiste européen. Certes, leurs prémisses étaient observables plus tôt. On peut, comme le fait le philosophe allemand Jurgen Habermas, y voir la fin du compromis établi après la Seconde Guerre mondiale sur la base de la défaite du fascisme ("L'unique évènement qui non seulement divise chronologiquement le siècle, mais encore représente une ligne de partage du point de vue économique, politique et surtout normatif $\left.{ }^{3} »\right)$, et donc étudier comment la révolution de 1989 n'a été qu'un point de retournement, l'aboutissement d'un long processus de déstabilisation. Dans tous les cas, la recherche de ses causes et de ses formes ne peut ignorer l'ampleur et la diversité de ces résultats. Ils invalident immédiatement toute vision «téléologique» ou «déterministe» qui expliquerait le processus par son objectif inéluctable (la démocratie).

«Transitologie » et « transformation »

5 C'est pourquoi la notion de transition avancée par la plupart des observateurs dans la foulée de la science politique anglo-saxonne ne nous paraît pas convaincante. Une nouvelle " discipline » a même été inventée à cet effet : la «transitologie ». Fondée sur les cas sud-européens (Espagne, Portugal) et latino-américains, elle a été conçue à la fin des années 1980 par Philippe Schmitter ${ }^{4}$. Elle a donné lieu à une abondante littérature analytique et critique $^{5}$. Elle propose, selon les auteurs, une définition plus ou moins large de la transition: soit le passage d'un régime à un autre, période généralement brève, soit pour ceux qui considèrent la « consolidation » incertaine, un processus plus long. Les auteurs penchent cependant pour la première option, certains considérant que l'organisation de deux élections libres et l'édiction de nouvelles règles suffisent à clore la transition ${ }^{6}$.

6 Les questions qui nous intéressent et que soulève la fin du communisme en Europe centrale et orientale sont d'une autre ampleur: comment interpréter la chaîne d'événements qui bouleverse l'Europe entre 1988 et 1991 ? Quelles sont les motivations des acteurs ? Maîtrisent-ils leurs choix ? Pourquoi l'effondrement à cette époque, et pas en 1956 ou en 1968 ou en 1980 ? Autant de questions pour lesquelles la " transitologie » est de peu de secours. Pas même lorsqu'elle invente une nouvelle discipline, la « consolidologie », pour comprendre les difficultés à " consolider » en une "routine » démocratique de nouvelles règles du jeu politique ${ }^{7}$. Le seul intérêt de cette démarche est de fixer un but - la démocratie - à un ensemble de conduites désordonnées, et de définir l'ordre à atteindre. L'accent mis sur les règles et les normes fournit une théorie à une conception du changement qui s'appuie sur les «forces de l'ordre" (les procédures, l'État, la bureaucratie...), espérant ainsi modifier les « conduites », voire les mentalités. Elle n'explique rien sur le plan analytique - l'évolution réelle est plus complexe et divergente; elle contribue plutôt à la consolidation d'un discours conservateur et téléologique sur l'histoire politique.

La transformation et ses incertitudes

7 Nous pouvons au contraire, à partir d'une approche sociologique et historique du politique (État, organisations, réseaux) et de l'action collective (mouvements sociaux, nationaux, etc.) comprendre l'enchaînement de crises et d'événements qui, en trois années et à partir d'un ensemble transnational (l'Europe centrale et orientale), ébranle le monde. Cette interprétation laisse un espace d'autonomie et de responsabilité aux acteurs du changement. Pour limitée que soit leur rationalité, ils peuvent orienter le 
mouvement par leur choix. Aussi préférons-nous à la notion de transition celle de «transformation » employée ${ }^{8}$ dans la lignée du livre fondateur de Karl Polanyi ${ }^{9}$. Elle présente l'avantage d'aborder le changement social comme un processus, un processus complexe aux multiples déterminations où les projets se heurtent, mais un processus construit. L'issue et la forme de ce processus ne sont pas acquises d'avance, elles dépendent de cette combinaison. Dans cette perspective, "les révolutions sont des formes spécifiques de transformation ${ }^{10}{ } »$.

8 En fait, en introduisant les concepts de «type d'État » (Pierre Birnbaum ${ }^{11}$, Charles Tilly ${ }^{12}$ ), « d'acteurs stratégiques » (Erhard Friedberg ${ }^{13}$, Alain Touraine ${ }^{14}$ ) et de «famille politique " (Stein Rokkan ${ }^{15}$ ), nous pouvons comprendre comment se constituent les acteurs dans le contexte particulier des régimes communistes d'Europe centrale et orientale, comment ils agissent et transforment leurs conceptions au cours des mobilisations et des crises. La prise en compte du passé dans ses diverses temporalités ne condamne ni à l'historicisme ni au relativisme culturel. Il s'agit plutôt de "rendre compte du sens que [les acteurs] investissent dans l'histoire, comme des contextes qui s'imposent à eux ${ }^{16}$ ». Ceci suppose une analyse fouillée de leurs conceptions politiques, de leurs représentations, des traditions qu'ils convoquent, de leur capacité d'invention, un travail de terrain et une vérification microsociologique de leurs pratiques.

Ces problèmes de méthode nous invitent enfin à une remarque sur l'incertitude de l'évolution historique. L'incertitude ne provient ni de l'enrayement de telle ou telle mécanique du fait de l'inexpérience ou de la maladresse de ses utilisateurs, ni même de l'irrationalité du choix des acteurs, mais de ce bricolage permanent entre des organisations qui se délitent, des identités multiples et mouvantes, des objectifs qui se dérobent et des valeurs instables. Un bricolage qui ne peut être étudié qu'ensuite, et qui ne produit pas toujours le meilleur. La fin du système communiste en Europe a sans doute libéré des millions de personnes du joug totalitaire, mais elle n'a pas été forcément «progressiste ». Elle a aussi créé de nouvelles conditions favorables pour la guerre, la misère, le crime contre l'humanité, les viols et les tortures sur le continent. Elle a jeté des millions de réfugiés sur les routes, et accru les tourments du monde. Si elle pousse les États et les peuples à s'associer, elle a entretenu aussi des haines. Il y avait de nombreuses incertitudes hier et il en reste encore beaucoup pour l'avenir.

La crise du système totalitaire

10 Nous partons, pour l'analyse de cette transformation, d'une définition du système politique communiste comme un système totalitaire. Cependant, l'État n'y a pas pour autant totalement liquidé et atomisé la société au point de nier toute vie autonome. L' homo sovieticus d'Alexandre Zinoviev ${ }^{17}$ n'a jamais existé. C'est pourquoi nous distinguons le projet totalitaire d'un parti qui s'empare de l'État et qui finit par se confondre avec lui ${ }^{18}$, de sa mise en œuvre dans une société historiquement construite. Le parti installe d'emblée l'État dans une relation conflictuelle avec les forces sociales qu'il est censé incarner. L'histoire ne s'arrête pas, au contraire, elle est façonnée par les hauts et les bas de cette relation. Tantôt l'ensemble paraît faire bloc, la société adhère à l'État, s'y assimile, tantôt des acteurs sociaux, même embryonnaires, résistent et des répressions de masse mobilisent les ressources modernes du contrôle, du crime et de l'embrigadement, tantôt des compromis partiels sont possibles et acceptés de part et d'autre.

11 De très nombreuses recherches historiques, faisant appel aux méthodes de la sociologie politique, de l'anthropologie, de l'histoire sociale ou de la sociologie de l'organisation, 
ont décrit et analysé ces conflits à différents moments et dans différents contextes géographiques tout au long du $\mathrm{xx}^{\mathrm{e}}$ siècle. Elles supposent une approche dynamique des systèmes totalitaires et de leurs transformations internes. Ici nous nous séparons des analyses «idéocratiques» qui finalement nient l'existence de rapports sociaux, surestiment la puissance du projet et l'achèvement de sa réalisation. Inversant la vulgate marxiste, elles font de l'idéologie une « infrastructure » au point, écrit Martin Malia, de « construire un monde où nous n'avons jamais eu affaire à une société, mais toujours à un régime, et à un régime idéocratique ${ }^{19}$ ». Nous nous référerons plutôt aux critères énoncés par Raymond Aron pour caractériser un régime totalitaire: le monopole de l'activité politique par un parti, l'autorité absolue de son idéologie, le monopole d'État des "moyens de forces et des moyens de persuasion ", l'étatisation de "la plupart des activités économiques" et toute "faute commise dans une activité économique est simultanément une faute idéologique ", ce qui aboutit à " une terreur à la fois policière et idéologique ${ }^{20}$ ». Raymond Aron définit un "type idéal» pour caractériser ce projet spécifique dans l'histoire des idées politiques.

Il serait illégitime de confondre intelligibilité et nécessité de la combinaison. On comprend que ces différents éléments soient réunis, mais le fait qu'ils l'ont été dans nombre de cas ne prouve pas qu'ils doivent toujours l'être et que tout parti monopolistique doive aboutir à cette sorte extrême de terreur ${ }^{21}$.

13 C'est justement cette variation qu'il est intéressant d'étudier dans le cas particulier des systèmes communistes d'Europe centrale et orientale. Les conditions de leur avènement sont diverses. Parfois, il est possible de suivre un processus révolutionnaire proche de celui constaté en Russie: la lutte de libération nationale structure un mouvement social, radical et armé, au sein duquel le parti communiste acquiert une position hégémonique (Albanie, Tchéchoslovaquie, Yougoslavie). Tout aussi radicaux et violents, mais en opposition avec les mouvements de libération nationale, sont les exemples de Pologne, Hongrie, Roumanie et Allemagne de l'Est, où l'occupation du territoire par l'armée soviétique devint le facteur décisif d'instauration d'un nouveau régime. Le cas polonais est sans doute le plus typique. La liquidation physique ou la déportation des combattants de la résistance nationale majoritaire non communiste (l'Armée du Pays), doublée d'une occupation minutieusement préparée avec un pouvoir civil (police politique) et militaire soviétique contrôlant chaque "voivodie libérée ", impose, après un simulacre d'élections, le régime communiste.

14 Mais dans tous les cas, le lien, qui s'établit entre l'État nouveau et la société qui sort de la guerre, demeure fragile. Il allie terreur et adhésion volontaire, selon des mécanismes subtils qui contiennent en eux-mêmes les germes de leur crise, et, on le sait maintenant, de l'effondrement des régimes totalitaires.

L'analyse de cette relation toujours conflictuelle, dans sa dynamique propre et ses interactions avec l'extérieur, devient un objet central de recherche. Elle met à jour ce que la sociologue polonaise Jadwiga Staniszkis appelle la «dialectique du socialisme réel » et inverse la perspective de ceux qui ne voient dans le totalitarisme qu'un monde clos, arrêté, un État-ogre vainqueur qui avale la société et la digère éternellement. Version pessimiste du projet totalement abouti à l'image du cauchemar orwelien, ce régime n'a pratiquement jamais été réalisé pas même dans l'URSS stalinienne de la pire époque. Les recherches les plus récentes sur le système stalinien montrent que, même à cette époque, la victoire et la domination d'un clan reposent sur une tension permanente entre le projet d'État à construire et la réalité des bouleversements 
institutionnels, économiques et sociaux ${ }^{22}$. Ils rejoignent en ceci les travaux pionniers de l'histoire sociale ou de l'analyse économique du système soviétique ${ }^{23}$. Le travail du chercheur peut alors se concentrer sur l'analyse des ressources mobilisées par l'État pour imposer et légitimer son projet, mais également sur la multitude des formes d'autonomie, même minuscules, dont disposent ou qu'inventent les acteurs sociaux. Les uns et les autres sont d'ailleurs traversés par des conflits tout aussi complexes.

Un enchaînement de crises politiques

La "chaîne d'événements ${ }^{24}$ » qui commence en Pologne, Hongrie et Yougoslavie en 1988, qui se poursuit avec la chute du mur de Berlin et des crises en chaîne à Prague, Bratislava, Bucarest, Vilnius et Belgrade se conclut sur trois grandes «nouveautés »: l'unification allemande, la dislocation de l'Union soviétique et dix années de guerre dans les Balkans. C'est en prenant en considération cet enchaînement supranational que nous discutons des causes et des formes de ces résultats.

Dans tous les cas, nous constatons une articulation entre des mouvements sociaux, des acteurs nationaux ou locaux, organisés au sein de l'état-parti ou indépendants, et les puissances voisines (principalement l'URSS, les États-Unis et l'Allemagne fédérale). Les mobilisations ne se dirigent pas toujours dans le sens de la démocratie, notamment en Yougoslavie. Elles invoquent des valeurs (la vérité historique, la liberté, les droits sociaux) ou des passions (le désir d'Europe, la haine ethnique, l'anticommunisme) ou des rêves (la solidarité universelle, la pureté nationale, le bonheur, la sécurité...), mais rarement des projets politiques clairs. L'articulation de ces mouvements avec les acteurs politiques et institutionnels a considérablement varié selon les conjonctures. En Pologne comme en Serbie, le mouvement a directement porté au pouvoir de nouvelles équipes, tandis qu'en Allemagne de l'Est et en Hongrie la disjonction fut plus grande. Les élites ouest-allemandes récupèrent à leur profit le soutien populaire dont bénéficiait le Neues Forum ${ }^{25}$, et en Hongrie, c'est la droite populiste qui s'installe au gouvernement, en maintenant dans l'opposition les dissidents libéraux et les réformateurs communistes. Deux évolutions analogues pourraient être constatées en Tchécoslovaquie ${ }^{26}$ et en Roumanie. Dans ce dernier cas le divorce donnera lieu à de violents affrontements en juillet $1990^{27}$.

L'initiative décisive durant les crises revient souvent à des acteurs nationaux, mais leurs projets (ou intention affichée) changent de nature durant le processus. Slobodan Milosevic conquiert l'appareil du parti sur une base conservatrice, communiste radicale (" la révolution antibureaucratique ») et recentralisatrice (le pluralisme sans partis, le " yougoslavisme unitaire »), puis il renonce à la Yougoslavie pour la "Grande Serbie ». En Pologne, les responsables de Solidarité n'envisageaient pas la prise du pouvoir politique et recherchaient un compromis avec les communistes. Puis, après les élections de juin, ils doivent rompre avec «l'autolimitation» et former un gouvernement. Une évolution identique est sensible chez les opposants hongrois. Quant aux animateurs des manifestations contestataires en Allemagne de l'Est, ils ne recherchent pas d'abord l'unité allemande, comme l'a fait une partie de la gauche de l'Allemagne fédérale ${ }^{28}$.

Les acteurs internationaux restent prudents, hésitants, conservateurs. Trois personnages s'imposent toutefois, pour leur ténacité et l'audace de leur entreprise. C'est d'abord le secrétaire général du PCUS, Mikhaïl Gorbatchev, qui se tient à sa stratégie de repli de l'empire et de construction d'une "maison commune » (fortement aidé économiquement par l'Occident, surtout l'Allemagne). Malgré son intrépidité dans 
le contexte de la "deuxième guerre froide » des années 1980, cette stratégie se révèle illusoire et se solde par une catastrophe. Les tentatives de reprise en main après novembre 1989, notamment en lançant les dernières interventions militaires soviétiques dans les Pays baltes et le Caucase, n'y peuvent rien. Mikhaïl Gorbatchev doit s'effacer. Le second héros de cette période est le chancelier de l'Allemagne fédérale, Helmut Kohl. Fidèle à la tradition de l'Ostpolitik initiée dans les années 1960, il privilégie d'abord "le changement par le rapprochement»: il ne met pas en cause l'existence de la RDA et semble de ce fait confirmer les desseins de Mikhail Gorbatchev, mais il ne perd jamais de vue l'unité allemande. Il n'hésite pas, par culture nationale et conviction européenne, à s'appuyer sur les mobilisations populaires pour transgresser le tabou. Le 28 novembre 1989, il sait prendre l'initiative décisive et tracer une orientation qui rompt le rapprochement tactique avec l'URSS. Sa victoire, moins d'un an plus tard, est à la mesure du dépit gorbatchévien. Elle fait de l'Allemagne un des acteurs centraux du rapprochement entre les deux Europe ${ }^{29}$. Enfin, il faut souligner le sens tactique de Slobodan Milosevic qui sait capter au profit de son pouvoir personnel les multiples mécontentements de l'après-Tito. À défaut d'une visée stratégique claire (d'abord la Yougoslavie, puis la Grande Serbie, puis son seul pouvoir...), il manœuvre avec brio entre les contradictions intérieures et les désaccords des grandes puissances internationales. Héritage perverti de la diplomatie de la « troisième voie » chère à Tito, le jeu international de Slobodan Milosevic lui permit de maintenir son clan au pouvoir sur la base d'une succession d'échecs militaires.

Pour discuter la causalité de ces résultats acquis en 1991, il nous paraît nécessaire de placer au centre de la réflexion la relation entre l'État et la mobilisation sociale : l'État comme centre du pouvoir politique devient le lieu et l'enjeu central des luttes sociales; la mobilisation appréhendée sous toutes ses formes, spontanées ou organisées, connaît de nombreuses modifications. Les fins et les moyens changent et interagissent.

21 Nous formons l'hypothèse que la manière dont cette relation se noue dans une conjoncture est fortement déterminée par un alliage subtil entre l'héritage acquis sur la longue durée et l'invention propre des acteurs devenus sujets. En l'occurrence la chute des régimes communistes aura été possible, à ce moment, de cette manière, et dans cette région justement parce que sont apparus des acteurs dotés certes d'une rationalité limitée, mais responsables de leurs choix. Quant à la forme des ruptures obtenues, du fait de la centralité de l'État dans l'objectif final et à cause de leur portée historique, nous la définirons comme une révolution européenne. Nous nous situerons ici dans la suite de Charles Tilly qui, à partir d'une définition « large » du phénomène ${ }^{30}$, pose deux questions pour apprécier une "issue révolutionnaire » : quels changements la crise étudiée introduit-elle dans la nature des États et dans la relation entre États? Quelle place ces changements occupent-ils dans les mutations politiques à long terme? L'articulation entre des États totalitaires en crise et les mobilisations sociales doit être conçue comme une interdépendance qui, dans la conjoncture de la fin des années 1980, fragilise totalement l'équilibre du système. Elle crée une opportunité politique.

23 Nous avons évoqué plus haut comment les contradictions structurelles du socialisme réel accumulaient les tensions, les déséquilibres et libéraient l'espace "gris » où pouvaient s'organiser des « résistances » les plus diverses, voire des acteurs sociaux et politiques. Nous avons également souligné comment les acteurs se construisaient une identité individuelle et collective en l'enracinant dans des traditions. Nous pouvons maintenant développer notre hypothèse en soulevant trois questions, plus précises. 
Pouvoir totalitaire et mobilisations collectives particulier des régimes communistes finissants? La plupart du temps, elles naissent sur des objectifs limités, rarement liés au politique. Ici, nous devons retenir provisoirement la distinction méthodologique énoncée par François Chazel ${ }^{31}$, entre le "conflit politique » sur lequel se concentre Charles Tilly ${ }^{32}$ et les "processus politiques » qu'il considère sur une plus vaste amplitude. Il cite à ce propos les travaux de Dong Mac Adam $^{33}$ sur le mouvement des droits civiques des Noirs américains.

Il met l'accent sur le «déroulement du processus » qui peut être très long, en distinguant deux situations : l'émergence du mouvement et son développement. Dans la première situation, «le degré de préparation organisationnelle de la collectivité de référence » apparaît comme " un facteur décisif pour l'émergence d'un mouvement protestataire», et dans la seconde, ce sont les "chances politiques (political opportunities) offertes aux membres d'un groupe potentiellement contestataire » qui favorisent le développement du mouvement social ${ }^{34}$.

Cette distinction est parfaitement confirmée par les trois « maillons faibles » à l'origine de l'enchaînement des crises en 1988-1989. Seule une analyse superficielle pourrait ignorer l'organisation d'oppositions en Pologne, Hongrie ou Yougoslavie, et l'affaiblissement des pouvoirs centraux (Jaruzelski, Kadar, Tito) accentué par la politique européenne de la Perestroika, qui constituent les chances politiques dont ils savent se saisir. La conjonction de ces conditions de base n'est présente, avec ce potentiel, que dans ces trois pays. En Allemagne de l'Est, en Tchécoslovaquie, en Roumanie ou en Albanie, le niveau d'organisation des groupes contestataires est beaucoup plus faible pour des raisons diverses liées à une plus grande efficacité du projet totalitaire de l'État-parti. En Albanie ${ }^{35}$ et en Roumanie ${ }^{36}$, il mobilise, en plus d'un encadrement policier de la population, une rhétorique nationaliste qui capte l'imaginaire national. En RDA et en Tchécoslovaquie, la répression également forte (rôle de la Stasi et de la «normalisation ») est couplée avec un consumérisme factice. Mais lorsque l'opportunité politique se présente en 1989, les petits groupes organisés se retrouvent à la tête de puissantes mobilisations.

Là encore, la "préparation organisationnelle » des années 1970 et 1980 sera décisive. En Tchécoslovaquie, malgré la répression des années 1970, une "société dissidente " était solidement enracinée à Prague, Brno et Bratislava. Elle ne comptait que quelques milliers d'activistes, mais agissait et parlait en écho d'une longue tradition de luttes démocratiques sous le communisme ${ }^{37}$ avant et après 1968. Les aspirations nationales et écologistes étaient également très présentes en Slovaquie. Vaclav Havel et ses amis sont portés au pouvoir par le mouvement et ils y restent, même si la majorité des nouveaux dirigeants est issue de milieux plus conformistes. En Allemagne de l'Est, au contraire, l'histoire plus récente, plus éclatée et plus marginale des groupes d'opposition, et leur dépendance maintenue à "l'identité est-allemande ", les laissaient sans force face aux puissants et riches appareils politiques débarqués d'Allemagne fédérale après la chute du mur. Ils seront balayés par la tourmente. Seuls se maintiendront, avec une légitimité ambiguë, les groupes issus des courants réformateurs de l'appareil de la SED. Les cas albanais et roumain confirment cette règle ${ }^{38}$. Le cas yougoslave est plus complexe, mais il confirme le modèle : les groupes nationalistes qui s'affirment dans les républiques au milieu des années 1980 sont tous issus des luttes contre le régime titiste en Serbie, Croatie ou Bosnie. Certains dirigeants avaient été emprisonnés dans les années 1960 ou 
1970. De même les oppositions démocratiques apparues dans les années 1960, en particulier autour du groupe Praxis, avaient connu des évolutions contradictoires avec de forts liens avec l'Ouest.

Ainsi, il serait tout à fait possible d'établir une sorte de géopolitique des sociétés dissidentes prises au sens large, dans les années 1960 à 1980, et d'y retrouver, presque mécaniquement, la géographie des crises qui secouent la région de 1988 à 1992. Toutefois, un troisième élément doit être pris en compte pour comprendre comment fonctionnent ces mobilisations sous le communisme. François Chazel poursuit le raisonnement de Doug Mac Adam en s'intéressant à la manière dont se construit l'identité collective de ces mobilisations. L'analyste du mouvement des Noirs américains évoquait une dimension "symbolique » et "cognitive", qui fait que "le mouvement lui-même doit être considéré comme une force autonome façonnant, jusqu'à un certain point, et dans certaines limites, son propre développement ${ }^{39}$ ». Il s'agit donc de comprendre comment cette dimension «culturelle» de la mobilisation s'établit, se métamorphose, agit en retour sur le mouvement lui-même.

Les conditions particulières des régimes communistes mettent en valeur des mécanismes moins visibles dans des régimes démocratiques où le débat et les identités politiques sont, a priori, plus encadrés. Quand il existe des partis, des médias et des débats d'idées plus ou moins libres, les clivages deviennent plus clairs, ils peuvent entrer dans des classifications plus traditionnelles (droite, gauche, libéral, conservateur, etc.). La production idéologique du socialisme réel, ses rituels, ses tentatives d'accaparer à son profit des traditions, le tout au service d'un projet totalitaire irréalisable, suscitent, en retour, des idéologies confuses, mélangées. L'absence de débat libre, la transmission orale pendant des décennies, par les familles ou les églises, des mémoires collectives ont entretenu un matériau culturel, symbolique et politique dont vont s'emparer ces mouvements ${ }^{40}$. La ritualisation par le pouvoir d'idées ou de symboles qui incarnent partout ailleurs la liberté et la démocratie (cas par exemple des fêtes du 8 mars et du 1er mai), les a dévalués.

Inversement se sont recyclés dans les discours d'opposition démocratique d'autres symboles ou d'autres idées que le monde entier considère relever de traditions réactionnaires et conservatrices. Quand en plus, entre les deux discours - le « rituel » et «l'authentique »- s'était durablement installé le souvenir interdit ou manipulé, ou récupéré d'un événement traumatique, le dialogue devenait impossible. Ceci pour souligner la complexité et l'hétérogénéité des références alternatives dont se sont emparés les mouvements sociaux qui deviennent en cette fin des années 1980, les premiers acteurs d'un changement radical.

31 Ce ne sont pas des acteurs mus par un intérêt commun dont ils auraient pris conscience et qu'ils sentiraient en péril : les motivations sont très diverses et hétérogènes au sein de la même société. Dans la plupart des cas, elles sont unifiées par des actes symboliques ou moraux à très faible portée politique: manifestations du souvenir, luttes économiques, peur de l'autre, fuite et migration, protestation contre la répression, concerts rock, défense de l'environnement, refus d'une humiliation locale, etc. La spontanéité des mouvements (qui n'est pas totale puisque existent des préparations organisationnelles) est accentuée par ces petits enjeux aux grandes conséquences. Les acteurs centraux, ceux que ces mouvements reconnaissent comme « leaders », sont-ils quand même dotés d'une « stratégie »? 

groupes politiques existent. Ils rassemblent quelques milliers de militants expérimentés, mais leurs projets sont généralement assez éloignés de ce qui va se dérouler effectivement. Au mieux, ils «accompagnent » le mouvement en cherchant empiriquement à l'orienter vers leurs perspectives. Il n'est donc pas possible de les appréhender comme une avant-garde, cette " conscience organisée » qu'avaient tenté d'incarner dans d'autres révolutions les théoriciens marxistes-léninistes. Ni au contraire, dans une logique utilitariste, comme des «free riders » ou des "passagers clandestins » qui auraient, à l'instar des dirigeants syndicaux britanniques, profité d'une action collective pour leur projet individuel (le pouvoir) ${ }^{41}$. En fait, ce sont ce qu'Erhard Friedberg appelle des "acteurs stratégiques", individuels ou collectifs, " porteurs de rationalité et donc capables de choix ».

ta sociologie des organisations facilite ici la compréhension de la façon dont se construit le mouvement, avec quels matériaux culturels, quels "agents", comment il évolue et réagit aux contraintes.

(teurs/agents sont rationnels et censés calculer leur conduite. Ceci n'exclut ni les émotions, ni l'intuition, ni le poids de l'histoire et/ou des institutions : la notion de rationalité utilisée ici intègre bien entendu toutes les limitations (cognitives, affectives, culturelles, idéologiques, etc.) que les travaux sur la théorie des choix ont mis en évidence ${ }^{42}$.

La notion de "rationalité limitée » permet d'évaluer, sans tomber dans le culturalisme ou la psychologie collective, les données dont disposent les acteurs et donc les "prémisses que le sujet accepte comme base de son choix ${ }^{43}$ ». Ces prémisses les dotent d'une "historicité» ou d'une «identité » qui se transforme à mesure que le mouvement se développe. Leur fin évolue au contact d'autres acteurs auxquels ils s'opposent, avec lesquels ils interagissent. Ils peuvent changer rapidement si ce jeu s'intensifie et s'accélère. Ce sont des acteurs mutants.

Trois éléments se combinent donc en un même processus mobilisateur : la préparation organisationnelle (qui facilite son émergence), l'opportunité politique (qui accélère son développement) et ses mutations identitaires (qui orientent ses fins). Ces éléments ne sont pas fixés a priori (l'analyse les met en évidence ex-post, dit Erhard Friedberg) mais leur combinaison dépend des «règles du jeu» qui prévalent au sein du système considéré.

Une révolution européenne

D'où notre deuxième question: comment les mobilisations affectent-elles l'équilibre d'un système dont nous avons constaté la complexité, cet échafaudage résultant de multiples compromis et rapports de force, et comment finalement naît l'opportunité d'une rupture radicale ? Ici notre refus de considérer les régimes totalitaires à l'image « idéocratique » d'un système de contrôle total et accompli, prend toute sa valeur. Si tel avait bien été le projet, il s'était immédiatement heurté à des résistances. Mais, contrairement aux régimes à dominante démocratique, les régimes communistes ne possédaient guère de "corps intermédiaires» capables d'amortir les chocs protestataires. Dans leur phase décadente, ils ont certes tenté de «déconcentrer » ou de " partager » les responsabilités, en faisant appel à des compromis avec les groupes sociaux ou communautés contestataires. Nulle part les résultats n'ont été concluants. On a vu, en revanche, les initiatives prises par le Centre se transformer et aboutir à des résultats inverses de ceux recherchés. D'où cet équilibre instable et ces multiples 
"scènes" où se «nouaient" des compromis. L'organisation sociale construite par le pouvoir totalitaire avait tendance à se relâcher et les règles formelles à se relativiser.

Ces transformations ont accentué l'écart entre les règles formelles et l'organisation vécue au point que l'émergence de mouvements sociaux a mis rapidement en péril l'ensemble de l'édifice. Ils se sont immédiatement heurtés au pouvoir central incarné par le parti unique et son "bureau politique ». Il y avait là une constante, sinon un anachronisme jamais remis en question par les dirigeants communistes. Ils ont été parfois prêts à d'importantes concessions, tolérant jusqu'à un "pluralisme bancal » (lame pluralism) selon l'expression de la sociologue polonaise, Jadwiga Staniszkis ${ }^{44}$. Mais jamais ils n'ont accepté un réel pluralisme et la séparation des pouvoirs exécutifs et législatifs. Ils ont à l'inverse fait inscrire dans la plupart des constitutions le "rôle dirigeant du parti » (réformes des années 1970), désignant ainsi la limite infranchissable. Des protestations dans plusieurs pays n'y ont rien fait. C'est d'ailleurs lorsque ce verrou a sauté, avec l'organisation d'élections libres en 1989-1990, que l'autorité centrale du parti s'est effondrée.

39 Ainsi, en maintenant coûte que coûte leur projet central, les dirigeants communistes, quelle que soit leur bonne volonté libérale, perdaient leur possibilité d'amortir le choc des protestations. La déliquescence de l'organisation et la dynamique propre aux mouvements qui se développent rapidement et sans contrôle, du fait de l'opportunité créée, aboutissent à un face à face fatal. Le pouvoir est faible. Il perd d'un seul coup sa légitimité et ses capacités d'intervenir. Ces moments deviennent révolutionnaires justement parce que le pouvoir communiste, ainsi dénudé, se trouve seul face à un contre-pouvoir de plus en plus légitimé. En capitulant (RDA, Tchécoslovaquie, Pays baltes), en se retirant (Pologne, Hongrie) ou en fuyant (Roumanie), il lâche le système qui s'effondre. L'opportunité politique se transforme en une rupture radicale, avec cette particularité notée plus haut, que les protagonistes eux-mêmes perdent confiance en leur projet (surtout les communistes polonais et hongrois). L'idéologie gorbatchévienne de rechange fait long feu. Seuls les appareils ou factions de la nomenklatura, qui réussissent à se reconstituer une identité plus ou moins légitime en s'appropriant les nationalismes locaux, peuvent reprendre partiellement pied (Roumanie, Bulgarie) ou conserver l'initiative en engageant une autre mutation (Serbie). Mais dans tous les cas, la rupture est nette avec le système communiste.

La compréhension de ce "jeu» entre les acteurs lors des crises conduit à deux conclusions complémentaires. La première insiste sur la place centrale du politique dans le processus de mobilisation. Nous rejoignons ici la perspective du «conflit politique » de Charles Tilly qui explique comment ces mobilisations peuvent trouver des alliés dans les gouvernements en place, voire être kidnappées par certaines factions. Mais plus fondamentalement, cette approche permet de "réintroduire l'État", son histoire et ses traditions comme variable centrale d'explication dans l'examen des ruptures constatées. Étudiant les grandes mobilisations européennes ${ }^{45}$, l'action des mouvements ouvriers ou les nationalismes des années $1930^{46}$, la sociologie historique du politique a démontré à quel point ces mobilisations "dépendent de l'entrecroisement de multiples clivages mais aussi et surtout du type d'État qui s'est construit dans chacune de ces sociétés: les profondes différences dans leur stratégie, leurs liens aux partis politiques, leur idéologie et d'une manière générale leur radicalisme s'expliquent en partie par leur rapport à l'État ${ }^{47} »$. Nous retrouvons cette même influence dans le cas qui nous occupe. 
41 L'opposition entre deux cultures politiques de longue durée éclaire à titre d'exemple ce point. Les traditions polonaise et serbe ont toutes les deux été marquées par une disparition plus ou moins longue de leurs États. La Pologne a été rayée de la carte à la suite de plusieurs partages entre les empires de 1792 à 1918, tandis que la Serbie a complètement disparu sous le joug ottoman de 1459 à 1817. L'une et l'autre ont conservé de ce passé une grande méfiance à l'égard du droit et des administrations imposées de l'extérieur. Mais outre que la rupture est plus profonde dans le cas serbe (quatre siècles au lieu d'un siècle et demi), les régimes déstructurés par les empires étaient très différents. La monarchie polonaise avait joué un rôle majeur dans la querelle des investitures ( $\mathrm{xI}^{\mathrm{e}}$ et $\mathrm{xII}^{\mathrm{e}}$ siècles) qui a fondé le droit romain et installé la dissociation entre les pouvoirs politique et religieux, à la source des États modernes. Et si la « république nobiliaire » de Pologne et de Lituanie n'était pas parvenue à imposer un État fort aux XVII ${ }^{e}$ et XVIII ${ }^{e}$ siècles, au point de disparaître, elle a légué une tradition dualiste et un imaginaire politique fondé sur l'organisation de la société civile face à l'État ${ }^{48}$. Au contraire, l'empire serbe de référence (Dusan 1331-1355) avait voulu copier le système byzantin fondé sur la fusion du religieux et du politique, gênant d'autant la naissance d'un État laïque moderne ${ }^{49}$. Ensuite, «le système ottoman du millet, en confiant la gestion des affaires civiles aux responsables ecclésiaux de chaque Église, dont l'Église serbe autocéphale (rétablie entre 1557-1766)» avait renforcé cette tendance. L'Église orthodoxe a "sauvé l'identité serbe » au prix d'un « repliement sur la tradition » et de «l'absence de légitimité de toute règle de droit stable ${ }^{50}$ ». Ce qui a légué un imaginaire politique communautaire où :

la violence est d'autant plus importante qu'en l'absence d'État reconnu comme légitime, un système anthropologique (un système de parenté et une ou des religions) réactive des signes identitaires (rites religieux, codes d'honneur ou la vendetta sert aussi à imposer à l'individu l'autorité du clan) pour sauvegarder une cohésion perçue comme menacée ${ }^{51}$.

Ces deux héritages opposés inspirent en grande partie la culture des élites contemporaines, face au politique. Les premiers se méfieront de l'État au point de rechercher le compromis, de privilégier l'organisation « par en bas » d'une alternative, mais ils le feront dans une perspective de modernisation politique (démocratie). Quant aux seconds, ils privilégieront le chef charismatique et le territoire comme cadre d'identification. Ils mobiliseront " par en haut » l'alternative nationaliste, « érigeront la nation serbe en État, en niant la réalité de la nation yougoslave délimitée par la citoyenneté ${ }^{52}$ ». Slobodan Milosevic construit l'État des Serbes en « tournant le dos à la modernisation » : «L'idée d'une Serbie unie, isolée, entourée d'une coalition hostile et menacée par un complot mondial, servait de prétexte pour contester la nécessité du changement dans le sens du pluralisme politique et pour défendre en fait le principe du parti unique ${ }^{53}$.»

43 Ce qui nous conduit à la deuxième conclusion. Le jeu entre les acteurs n'est ni gratuit ni vide. De grandes options les séparent, leurs orientations ne sont pas équivalentes. Ici se pose la question plus générale des «conceptions politiques » ou visions du monde des acteurs ${ }^{54}$ et de leur influence sur le cours des événements. Qu'elles changent au cours de la mobilisation est un fait déjà noté, mais elles le font dans le cadre d'une tradition. Une approche purement transactionnelle des relations entre acteurs ne nous convient pas. Nous avons au contraire plusieurs fois souligné comment les uns et les autres construisaient leurs représentations, leurs légitimités et leurs identités, à mesure que se développait le processus. Cette mutation permanente qui interagit sur le mouvement 
collectif, ne peut fonctionner que si elle s'intègre à des cultures politiques transmises par les traditions. Il n'y a pas de sujet politique vierge.

D'où l'importance des conceptions véhiculées par les acteurs. Pour en rester à notre exemple, les élites serbes et polonaises qui prennent le pouvoir (ou le conservent) après 1989, ne relèvent pas de la même "famille » politique. Les premières se placent dans une tradition autoritaire, centralisatrice et unitaire, en faisant appel à des méthodes populistes, tandis que les secondes se réfèrent explicitement et en pratique à une orientation libérale, démocratique et décentralisatrice. Sur ce point, nous retrouvons la théorie multidimensionnelle des familles politiques de Stein Rokkan ${ }^{55}$, d'une grande pertinence pour comprendre la formation des principaux clivages avant et après la chute du communisme ${ }^{56}$. Cette théorie tente en effet une explication génétique ${ }^{57}$ à partir des grandes contradictions qui structurent les sociétés. Mais elle se refuse à réduire ces familles aux reflets d'intérêts sociaux ou de lobbies. Une approche sociologique et historique permet de comprendre comment ces contradictions se transforment en conflits, puis en clivages ${ }^{58}$, structurant la vie politique. Elles donnent un caractère fondateur aux grands moments de polarisation politique (et de traumatisme social) comme les révolutions.

Dans le cas de la chaîne d'événements (1988-1992) qui nous occupe, cette remarque devient fondamentale pour identifier l'origine de la bifurcation entre les deux orientations maintes fois soulignées : la démocratie ou la guerre. Le jeu complexe entre les acteurs n'est pas seulement une question de rapport de force, ou une transaction d'intérêts ou de pouvoirs. La direction générale du processus est également déterminée par les conceptions politiques des acteurs qui se réfèrent à ces grands clivages. Les uns s'engagent résolument sur une voie démocratique et libérale, les autres, par la voix des élites au pouvoir, optent pour la corruption, le crime et la guerre. Ceci permet de mieux circonscrire les responsabilités individuelles et collectives. Il n'y a pas de fatalité, dans un sens comme dans l'autre.

Ainsi, après avoir étudié comment se développaient les mobilisations politiques et comment elles pouvaient créer l'opportunité d'une rupture radicale, une troisième question s'impose : quelle est la nature de cette rupture ? S'agit-il d'une révolution?

La notion de « révolution » n'a pas toujours bonne presse en science politique ${ }^{59}$. En plus elle a été en grande partie polluée par le modèle de la "table rase » des bolcheviks. Pourtant, la question mérite d'être posée. Il n'est pas forcément nécessaire d'inventer une théorie particulière, pour comprendre la portée historique de cette chaîne d'événements qui, aujourd'hui plus personne ne le conteste, a bouleversé l'Europe et l'ordre mondial. Il revient à Charles Tilly d'avoir tenté de mettre de l'ordre dans les concepts pour dégager une définition large de la révolution européenne. Il en étudie de manière convaincante les cheminements sur cinq siècles.

En partant des changements que la crise considérée a introduit dans la nature de l'État et la relation entre les États, et en appréciantleurs conséquences dans les mutations politiques à long terme, il retient trois "éléments constitutifs" d'une révolution : «Deux blocs politiques ou davantage disposant d'un soutien important " sont face à face, "des prétentions incompatibles à contrôler l'État les opposent", et la crise se solde par un «transfert du pouvoir d'État ». Pour Charles Tilly :

un cycle révolutionnaire complet va donc de la disparition de la souveraineté et de l'hégémonie [des autorités en place] à leur rétablissement sous une direction nouvelle, via une période de lutte. Le cours suivi par la lutte et par le changement 
entre l'ouverture et la clôture de la période de souveraineté multiple constitue le processus révolutionnaire ${ }^{60}$.

situations, avec cette particularité en Pologne et en Hongrie d'une mobilisation de concurrents au pouvoir en place qui ne se présentent pas d'emblée comme des prétendants au "contrôle exclusif de l'État». Ils avancent avec un programme qui recherche le compromis («table ronde») mais, la mobilisation sociale et la polarisation politique qu'elle provoque emporte, comme nous l'avons vu, ces concurrents au-delà de leurs désirs. Dans le cas serbe, le mécanisme est plus classique, la stratégie de Slobodan Milosevic vise consciemment le contrôle de l'État: elle combine une lutte au sein de 
l'appareil (1987-1989) et une mobilisation du "peuple serbe" hors des cadres traditionnels (1988-1990), pour rallier à ses prétentions l'ensemble des mécontents. En Allemagne de l'Est, en Tchécoslovaquie, en Roumanie, dans les États baltes et même en Albanie, on retrouve avec des mobilisations plus ou moins fortes et face à des pouvoirs plus ou moins résistants ou divisés, le même enchaînement.

"L'issue révolutionnaire » dépend principalement de la capacité des prétendants à maîtriser la violence légitime. Revenant sur les facteurs qui contribuent à la victoire des révolutions étudiées sur un demi-millénaire, Charles Tilly constate :

L'organisation de la force armée a été la vraie médiatrice entre la situation révolutionnaire et l'issue révolutionnaire. [Il insiste] Pas un seul [opposant] n'a pris le pouvoir d'État sans s'être préalablement assuré le contrôle effectif d'une force militaire.

Dès lors, l'issue résulte de quatre éléments :

1. Défection des membres du système politique.

2. Acquisition d'une force armée par les coalitions révolutionnaires.

3. Neutralisation ou défection des forces armées du régime.

4. Contrôle de l'appareil d'État par les membres d'une coalition révolutionnaire.

Quatre éléments qui se combinent un peu différemment selon les situations. En Pologne, Hongrie, RDA et Tchécoslovaquie, le ralliement au processus d'une partie de l'appareil du pouvoir (les "réformateurs») et le laisser-faire de l'Union soviétique dirigée par Mikhaïl Gorbatchev, neutralisent les forces de répression qui basculent du côté des opposants légitimés par des scrutins aux accents de plébiscites. En Roumanie, une fraction de l'armée et de la Securitate se range au côté des insurgés et légitime les postulants au contrôle exclusif de l'État en se retournant au besoin contre une partie des «révolutionnaires» (juillet 1990). En Serbie, le clan Milosevic berne l'armée fédérale en se présentant comme un partisan du yougoslavisme, la réorganise et entreprend une "serbisation " par la guerre. Dans les Pays baltes, lorsque le pouvoir fédéral soviétique tente, à Vilnius en janvier 1990 notamment, de réprimer la mobilisation nationale, une partie de la troupe (y compris les régiments d'élite!) se rallie aux manifestants.

Ainsi, dans le détail et selon un enchaînement dont les acteurs, à travers leurs projets ou leurs rêves, étaient loin de prévoir la fin constatée, la " grande secousse » de 1989 est devenue la dernière grande révolution $\mathrm{du} \mathrm{xx}^{\mathrm{e}}$ siècle. Elle a ébranlé le monde et beaucoup de grands esprits.

\section{NOTES}

1. Voir C.-P. David, La Guerre et la Paix, approches contemporaines de la sécurité et de la stratégie, Paris, Presses de la FNSP, 2000, p. 60.

2. B. Badie, «De la souveraineté à la capacité de l'État », in M.-C. Smouts (ed.), Les nouvelles relations internationales, Paris, Presses de la FNSP, 1998, p. 51-52. 
3. J. Habermas, Après l'État-nation, Paris, Fayard, 2000, p. 23.

4. G. O. Donnel, Ph. Schmitter, Transition. From authoritarian rules : Prospect for Democracy, Tentative conclusions about uncertain Democraties, Baltimore, John Hopkins University Press, 1986. Voir aussi J. J. Linz et A. Stepan, Problem of Democratic Transition and Consolidation, Baltimore and London, 1996.

5. Pour une vue d'ensemble voir J.-M. De Waele, « Les théories de la transition à l'épreuve de la démocratisation en Europe centrale et orientale ", in P. Delwit, J.-M. De Waele, La démocratisation en Europe centrale, Paris, L'Harmattan, 1998, p. 30 et sq. ; et "Les transitions démocratiques, regards sur l'état de la "transitologie" ", dossier de la Revue Française de Science Politique, Paris, vol. 50, n 4-5, août-octobre 2000.

6. J. Linz, A. Przeworski, cités par P. Delwit, J.-M. De Waele, 1998, p. 32.

7. Ibid., p. 619.

8. Voir H.-J. Wagener, «La transformation : un cadre historique et théorique », Revue d'Études comparatives Est-Ouest, Paris, CNRS, vol. 29, n 4, 1999, p. 5-21.

9. K. Polanyi, La grande transformation aux origines politiques et économiques de notre temps, Paris, Gallimard, 1983.

10. Ibid., p. 8.

11. B. Badie, P. Birnbaum, La sociologie de l'État, Paris, Grasset, 1982.

12. C. Tilly, Contraintes et capital dans la formation de l'Europe, Paris, Aubier, 1992.

13. E. Friedberg, « Organisation et action collective», in F. Chazel (ed.), Actions collectives et mouvements sociaux, Paris, Puf, 1993.

14. A. Touraine, Le retour de l'acteur, Paris, Fayard, 1984.

15. S. Rokkan, Citizens, Elections, Parties, Oslo, Oslo University Press, 1970.

16. Y. Deloye, Sociologie historique du politique, Paris, La Découverte, 1995, p. 26. Voir aussi le numéro spécial de Revue internationale des sciences sociales, « La sociologie historique, débat sur les méthodes », Paris, août 1992.

17. A. Zinoviev, Homo Sovieticus, Paris, Julliard, 1983.

18. Le projet du « Peuple-Un » tel qu'analysé par H. Arendt, Le système totalitaire, Paris, Seuil, 1977.

19. M. Malia, La tragédie soviétique, Paris, Seuil, 1995, p. 19 ; voir également M. Malia, Comprendre la révolution russe, Paris, Seuil, 1980, et pour une inspiration plus ancienne, J. L. Talmon, «Les origines de la démocratie totalitaire » (1952), in E. Traverso (ed.) Le totalitarisme, textes choisis, Paris, Points Seuil, 2001, p. 600 et sq.

20. R. Aron, Démocratie et totalitarisme, Paris, Gallimard, 1965, p. 284-285.

21. Ibid., p. 285. C. J. Friedrich et Z. K. Brzezinski en distingue six, en faisant de l'existence d'une police politique secrète un critère à part. Cf. Totalitarism Dictatorship and Autocracy (1956), Cambridge University Press, 1965.

22. Voir notamment $\mathrm{N}$. Werth, «Staline et son système dans les années 1930 », in $\mathrm{H}$. Rousso (dir.), Stalinisme et Nazisme, Bruxelles, Édition Complexe, 1999, p. 50.

23. M. Lewin, La formation du système soviétique, Paris, Gallimard, 1989 ; B. Kerblay, La société soviétique contemporaine, Paris, Armand Colin, 1977.

24. La notion d'événement employée ici a été définie par P. Nora, «Le retour de l'événement ", Faire l'Histoire, tome 1, Paris, 1974.

25. F. Bafoil, Règles et conflits sociaux en Allemagne et en Pologne postcommunistes, Paris, L'Harmattan, 1997, p. 42 et sq.

26. J.-Y. Potel, Les cent portes en Europe centrale et orientale, Paris, Éditions de l'Atelier, 1998, p. 247. 
27. Ibid., p. 252 et sq, et J.-Y. Potel, Quand le soleil se couche à l'Est, La Tour d'Aigues, Éditions de l'Aube, 1995, p. 234-243.

28. Voir par exemple le roman de G. Grass, Toute une histoire, Paris, Seuil, 1997.

29. Et non une nouvelle puissance impériale ! Voir sur ce rôle européen de l'Allemagne le dossier « l'Allemagne et l'Europe centrale à l'heure de la réconciliation ", Revue d'Études comparatives Est-Ouest, Paris, CNRS, vol. 31, n 1, mars 2000.

30. C. Tilly, Les révolutions européennes (1492-1992), Paris, Seuil, 1993, p. 24-27.

31. F. Chazel, « La place du politique dans les mobilisations contestataires », in F. Chazel (ed.), Actions collectives et mouvements sociaux, Paris, Puf, 1993, p. 151.

32. C. Tilly, From Mobilization to Revolution, Reading, 1978, et Les révolutions européennes (1492-1992), 1993, op. cit.

33. D. Mac Adam, Political Process and the Developement of Black Insurgency, Chicago, 1982.

34. F. Chazel, op. cit., 1993, p. 152.

35. E. et J.-P. Champseix, 57 Boulevard Staline, Paris, La Découverte, 1990, et A. Fuga, L'Albanie entre la pensée totalitaire et la raison fragmentaire, Paris, L'Harmattan, 1998.

36. Voir « Roumanie, crise et répression », L'Alternative, Paris, 1983.

37. Pour une étude de cette dissidence voir A. Madelin, Cahiers du CEFRES, Prague, 1995, et M. Novak, «L'influence de la dissidence dans la décomposition du régime communiste ", in J. Sémelin, La liberté au bout des ondes. Du coup de Prague à la chute du mur de Berlin, Paris, Belfond, 1997.

38. Sur l'évolution de l'opposition roumaine en 1990, voir M. Berindei, A. Combes, A. Planche, Roumanie, le livre blanc, Paris, La Découverte, 1990.

39. F. Chazel, op. cit., 1993, p. 152-153.

40. J.-Y. Potel, « La manipulation politique des mémoires », Historiens et Géographes, Paris, juin 2001.

41. M. Olson, Logique de l'action collective, Paris, Puf, 1978, p. 98.

42. E. Friedberg, « Organisation et action collective », in F. Chazel, op. cit., 1993, p. 240.

43. H. A. Simon, Administration et processus de décision, Paris, Economica, 1983, p. 72.

44. J. Staniszkis, Pologne : la révolution auto limitée, Paris, Puf, 1982, p. 162.

45. C. Tilly, From Mobilisation to Revolution, op. cit., T. Scockpol, États et révolutions sociales, Paris, Fayard, 1985.

46. P. Birnbaum, Le peuple et les gros, Paris, Grasset, 1979 ; La France et les Français, Paris, Seuil, 1993.

47. P. Birnbaum, « Mouvements sociaux et type d'États », in F. Chazel, op. cit., p. 167.

48. J.-Y. Potel, « Solidarité et les traditions politiques polonaises », in Quand le soleil..., op. cit., 1995, p. 160-164.

49. B. Badie, Culture et politique, Paris, Economica, 1983.

50. J. Krulik, « Réflexions sur la singularité serbe », Le Débat n 107, Paris, 1999, p. 98.

51. Ibid., p. 100.

52. O. L. Kubli, Du nationalisme yougoslave aux nationalismes post-yougoslaves, Paris, L'Harmattan, 1998, p. 198.

53. L. Petrovic, « Le dos tourné à la modernisation », in N. Popov (ed.) Radioscopie d'un nationalisme, Paris, 1998, p. 127.

54. Voir F. Châtelet, E. Pisier, Les conceptions politiques du $x x^{e}$ siècle, Paris, Puf 1983, p. 10-13.

55. S. Rokkan, Citizens, Elections, Parties, Oslo University Press, 1970 ; D.-L. Seiler, Partis et familles politiques, Paris, Puf, 1980. 
56. J.-Y. Potel, « Les recompositions politiques », in M. Fybres (ed.), La nouvelle Europe centrale, Paris, La Découverte, 1998.

57. P. Bréchon, Les partis politiques, Paris, Montchrestien, 1999, p. 66.

58. Qu'on ne doit pas interpréter automatiquement comme des oppositions de classes sociales.

59. Voir toutefois le numéro spécial de la Revue Française de Sociologie, Paris, 1989.

60. C. Tilly, Les révolutions européennes, op. cit., p. 31.

61. Ibid., p. 41.

62. Ibid., p. 363.

63. Voir le tableau, ibid., p. 364.

64. Ibid., p. 373-374.

\section{RÉSUMÉS}

Yves Potel souligne la variation des évolutions particulières des systèmes communistes d'Europe centrale et orientale, de leur avènement à leur disparition; il analyse la relation toujours conflictuelle entre les nouveaux États à construire et les sociétés communistes, en plein bouleversement institutionnel, économique et social, aux dynamiques propres, en interaction plus ou moins forte avec l'extérieur et qui ont toutes forgé, à des degrés divers, des formes d'autonomie ou de résistance. La mobilisation sociale face à l'État différencie les voies de sortie du communisme. Elle est aussi fonction de l'histoire politique propre de chaque société et de leurs capacités à inventer des voies de sortie spécifiques (comme Solidarité). Cette histoire influe sur la forme des ruptures obtenues qui jouent un rôle dans les mutations politiques observables. La fragilisation du système par ses contradictions structurelles a créé l'opportunité politique de poches diverses de résistance, souvent capables de s'articuler sur la construction d'identités collectives ou individuelles.

Yves Potel. The end of communism. The author underlines the variations in the particular evolutions of communist systems in Central and Eastern Europe, from their appearance to their end. He analyses the always conflictual relationship between the new states about to be built and the communist societies which have all evolved in varying degrees some forms of autonomy or resistance. Social mobilisation against the State has produced different ways out of communism. This mobilisation also depends on the particular history of each society and its capacity of inventing specific means of exit (such as Solidarity). This history has influenced the form of the breaks which have taken place and play a role in the political mutations observed. He insists on the fact that the fragilisation of the system through its structural contradictions has created a political opportunity for various pockets of resistance, often capable of linking with the construction of collective or individual identities. 
AUTEUR

JEAN-YVES POTEL

Institut d'études européennes, université de Paris-VIII, Dr hab. en science politique, jypotel@ipgate.pl 